\title{
Proficient routing by adroit algorithm in 5G-Cloud-VMesh network
}

\author{
P. Dharanyadevi ${ }^{*}$ and K. Venkatalakshmi
}

\begin{abstract}
$5 \mathrm{G}-$ Cloud-VMesh network ( $5 \mathrm{CVN})$ is nascent as an economical alternative for building federated and community networks. In 5 G milieu, the Aggregator Node B (ANB) is the base station with enhanced computing capabilities and storage space. In this paper, an integration of 5G-cloud-VMesh network is initiated. In this paper, we also propose a new routing algorithm for $5 \mathrm{CVN}$, dubbed as $5 \mathrm{CVN}$-adroit algorithm. The proposed $5 \mathrm{CVN}$-adroit algorithm chooses an optimal gateway and ANB in the network layer to route the request/response from and to the service requesters and the cloud using 5G technologies. This paper endows with the network architecture and main technical features such as interconnection, integration, and intelligence of $5 \mathrm{CVN}$-adroit algorithm. The simulation study shows that the $5 \mathrm{CVN}$-adroit algorithm augments the routing issues such as delay, congestion, and deployment cost and it is justified based on the packet delivery ratio, average response time, routing overhead ratio, and packet collision ratio.
\end{abstract}

Keyword: 5G, Vehicular network, Mesh network, Cloud, Routing, Performance

\section{Introduction}

Vehicular Ad hoc Networks (VANET) are pervasive, ubiquitous, and infrastructure-free without any centralized authority. VANET is a decentralized network where each node is proficient to forward data packets between the nodes dynamically [1-4]. In VANET, a node can be bus, tram, subway, train, or taxi. Vehicles get a broadband wireless access by using heterogeneous wireless technologies, namely IEEE 802.11, IEEE 802.16 (WMAN, WiMax), 3GPP Universal Mobile Telecommunications System (UMTS), and Long-Term Evolution. A wireless mesh network (WMN) is a multi-hop communication network in which the nodes are self-organized (i.e., without a central coordinator) [5]. VMesh is a hybrid network, which combines the features of VANET and WMN. VMesh network is the communication environment between the vehicles made up of radio nodes organized in a mesh topology. VMesh generates a loop among the moving vehicle. In the loop, we can add, update, or remove the nodes [6]. Vehicles are becoming more sophisticated with powerful onboard computing capabilities such as tons of on-board storage and short- or medium-range wireless transceiver

\footnotetext{
* Correspondence: dharanyadevi@gmail.com

${ }^{1}$ Information and Communication Engineering, Anna University, Chennai, India

Full list of author information is available at the end of the article
}

with no power limitations. Advancement in wireless networks crafts the access to the Internet from vehicles. The high-flying industries and governments such as Audi, BMW, Daimler-Chrysler, and Toyota have commenced important research for vehicle to vehicle communications. California Partners for Advanced Transit and Highways, Crash Avoidance Metrics Partnership, FleetNet, Advanced Driver Assistance Systems, Chauffeur in EU, CarTALK 2000, and DEMO 2000 are petty distinguished research, which are moving towards the realization of intellectual transport services.

With surging vehicle Internet services, VMesh network is facing unique opportunities and challenges. To coup the rapid development and demands due to the multimedia services, social networks, and entertainments, it is far from enough to only expand and upgrade current networks, which results in a substantial increase in CAPital EXpenditure (CAPEX) and OPerating EXpenditure (OPEX) without reasonable investment returns. Therefore, in order to offer a High-Quality of Experience (HQoE) for vehicle users with affordable costs, new network architecture is designed to enable sustainable network evolution to the $5 \mathrm{G}$. Till now, much attention has been paid to emerging $5 \mathrm{G}$ technologies ranging from information processing to networking techniques. Most of the focus has been on further improving radio access efficiency through advanced signal

\section{Springer}


processing, e.g., cooperative relaying, massive MIMO, and millimeter-wave communications [7-13]. On the other hand, the existing shaft-like network structure with centralized data processing is not suitable for flexible and diverse VANET Internet services, which undoubtedly restricts the service provisioning ability of the VMesh network. Hence, designing new network architecture becomes imperative. This motivates us to propose a new architecture for VMesh network with the integration of cloud and $5 \mathrm{G}$ technologies. However, there is still much work that needs to be done to improve the $5 \mathrm{CVN}$ architecture with good backward compatibility. This paper emphasizes how to maximize the utilization of resource without delay and congestion and to smoothly evolve towards converged $5 \mathrm{CVN}$.

The motivations of the paper are as follows, nowadays, users of all ages want to share and get the information at anytime and anywhere via vehicle Internet service. Also, the growth of the Internet-of-Things (IoTs) has had serious effects in the VANET performance. It is expected that by the year 2020, average user data rates will increase from around $20 \mathrm{MB} / \mathrm{s}$ to $1 \mathrm{~GB} / \mathrm{s}$, and connection density will also increase from 140,000 connections per square kilometers to 6 million connections per square kilometers. Moreover, new applications such as the Internet-of-Vehicles are envisioned for 5G, which will demand much shorter latency from the underlying network. Considering the existing network infrastructure, telecommunication operators need to invest heavily on both network maintenance and upgrade to support ever increasing capacity requirements. However, revenues from increased data traffic cannot keep up with the investment. How to reduce CAPEX/OPEX and create new revenue flows are crucial issues facing telecommunications operators. The proposed $5 \mathrm{CVN}$-adroit algorithm may perhaps solve the above issues.

In this paper, an integration of 5G-cloud-VMesh network is introduced. The envisioned architecture shall enable data access from the vehicles, anytime and anywhere. In particular, the integration of IEEE 802.11-based multihop VANETs with $5 \mathrm{G}$ shall contribute to the evolution of beyond $4 \mathrm{G}$ wireless communication systems. The proposed $5 \mathrm{CVN}$-adroit algorithm addresses the choosing of an optimal gateway and Aggregator Node B (ANB) in the network layer from and to the service requesters and the cloud using 5G technologies. 5CVN-adroit algorithm consists of two key processes such as metric-based gateway selection and metric-based ANB selection. In the majority of the existing research, gateways are considered static and deployed on the road at fixed distances from each other, depending on their wireless transmission range, which makes the deployment costlier. Moreover, the multi-hop and dynamic nature of VANET communication impacts the stability of the links to these gateways. Furthermore, as these gateways are fixed, the discovery and routing processes are mainly pro-active. Although the pro-active routing mechanisms reduce delay, they increase the frequent changes in the predefined routing tables of vehicles and routing overhead. To deal with these limitations, this paper defines the concept of vehicle gateways. Any vehicle in the VMesh network can act as a gateway. A gateway refers to the dual-interfaced vehicle that relays the packet from sources to the $5 \mathrm{G}$ backhaul network. It is enabled with dual interfaces of IEEE 802.11p and the 5G backhaul networks. The major challenge is to amalgamate these two network interfaces on a gateway, as they lie in two different spectrum regions. The next challenge is to choose a minimum number of optimal gateways using relevant metrics. There could be several nodes in the VMesh network, which possess the important criterion and required metric information, and hence, the qualification to serve as gateways. The focus of this paper is on defining a mechanism that chooses a minimum number of optimal gateways, at an instance, so as to avoid the bottleneck at the $5 \mathrm{G}$ backhaul network. With this regard, as pro-active gateway discovery reduces delay and reactive discovery reduces routing overhead, this paper envisages a hybrid gateway discovery mechanism for VMesh network, combining the pros of both pro-active and reactive concepts.

For example, network statistics show that $70 \%$ of data traffic is carried by $30 \%$ of base stations. To solve the above issue, we introduced ANB pool; a large number of ANBs can form an "ANB pool" with aggregated processing capabilities to serve the traffic requirements of the coverage area. For instance, there are certain regions with heavy traffic loads and certain ANB are overloaded. In such cases, ANB selection has been introduced to select the optimal ANB among the ANB pool. In the majority of the existing research, conventional centralized cloud is considered. However, the conventional centralized cloud may result in a high delay of offloading data delivery and in congestion of backhaul due to the transmission of the offloaded data. The perceived delay caused by the centralized cloud can be minimized by adopting a distributed cloud deployed over ANB of cellular network.

Our paper deals with the above-identified issues by the following key contributions such as:

- Our first goal is to propose a heterogeneous architecture combining IEEE 802.11p-based VMesh, cloud, and 5G. Furthermore, to make the vehicles prominently use the advantages of $5 \mathrm{G}$ to progress and expand their utility, we tested the proposed heterogeneous architecture under a realistic scenario where we consolidate real-world road topology generated by the microscopic mobility model provided by SUMO. We analyze $5 \mathrm{CVN}$ network topology characteristics over a 
huge scale urban area using a real vehicle mobility model.

- Our second goal is to develop a new routing algorithm (5CVN-adroit algorithm). The $5 \mathrm{CVN}$ adroit algorithm discovers an optimal gateway and ANB in order to route the service request from the service requester to the cloud. The main purpose of gateway selection in the $5 \mathrm{CVN}$ is to permit only a minimum number of vehicles to communicate with the ANB. Certainly, if all vehicles in a 5G VMesh network directly communicate with the ANB, it results in a bottleneck at the ANB. The rationale of ANB selection is to avoid the data traffic at the ANB.

- Our third goal is to analyze the performance of the proposed $5 \mathrm{CVN}$-adroit algorithm over various performance metrics, including packet delivery ratio, average response time, routing overhead ratio, and packet collision ratio.

The rest of this article is organized as follows. In Section 2, we discuss the proposed 5CVN architecture and its main features. In Section 3, we describe the 5CVNadroit algorithm. In Section 4, we discuss and analysis the performance of the $5 \mathrm{CVN}$-adroit algorithm. Finally, in
Section 5, we present our conclusions and a description of future work that needs to be done.

\section{Network architecture of 5CVN}

To facilitate the flexible network control and reduce the network capital and operational expenditures, the complimentary concepts, $5 \mathrm{G}$ software-defined networking (SDN) components, and cloud have been integrated with VMesh networks. Through the proper cooperation between newly developed $5 \mathrm{CVN}$ and radio access network, vehicle user can enjoy a much better quality of experience with lower access delay and congestion. 5G SDN is fast evolving and becoming the focal point and a growth area in networking. 5G SDN is a new paradigm in networking, e.g., data and control plane separation, globally optimized network management, and centralized network controller [14-16].

Figure 1 illustrates the $5 \mathrm{CVN}$ network architecture. The $5 \mathrm{CVN}$ is heterogeneous and IP-based model with relays. The architecture includes a number of independent vehicles with the autonomous radio access technologies, ANB, MME, Serving Gateway (SG)/Gateway GPRS Support Node (GGSN), SDN controller, and cloud server. VMesh network user's vehicle may have any technology provisions.

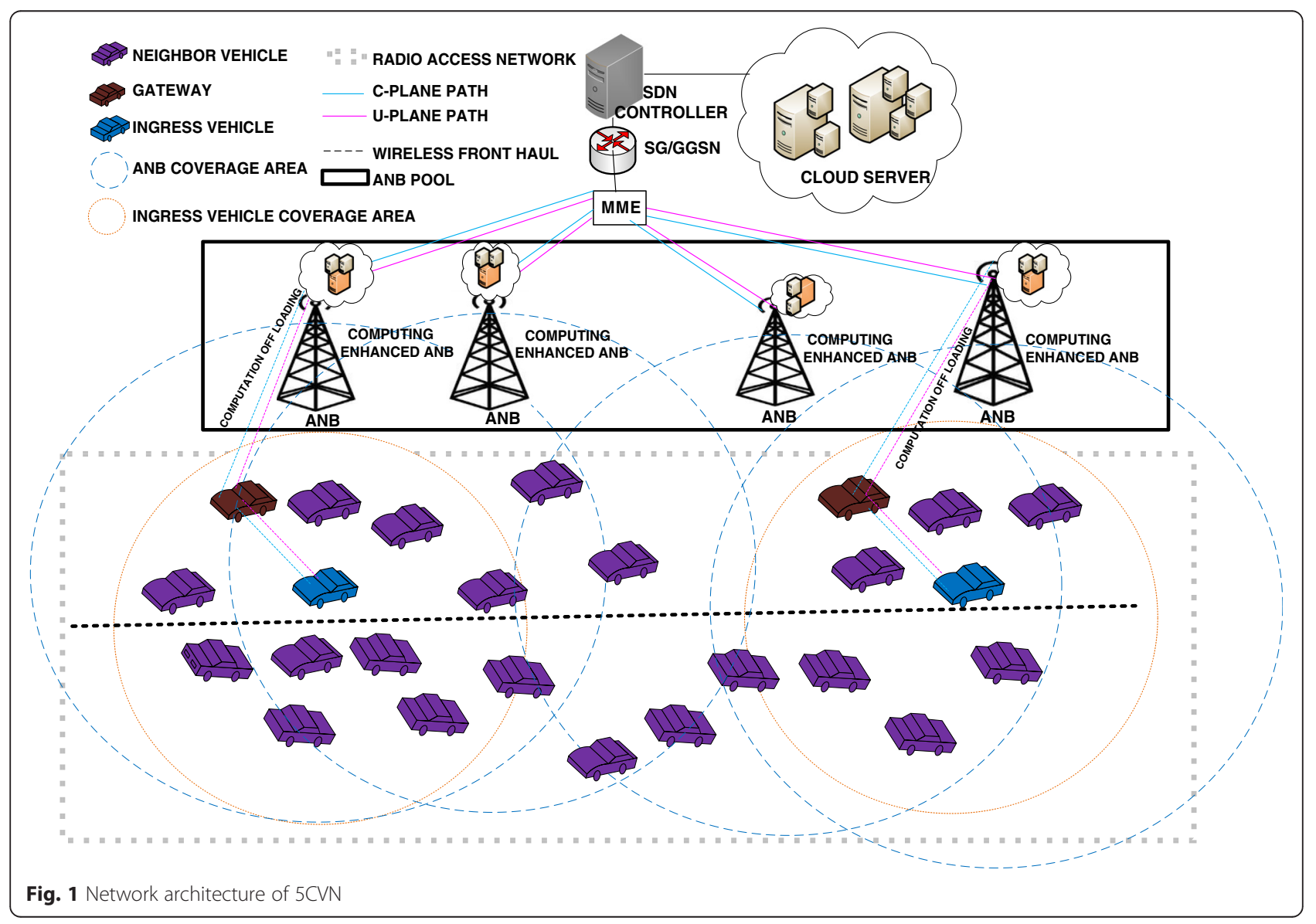


ANB has local cloud and it also has a computing capability for offloading a computation from the vehicle. The local cloud consists of the service which the user opts often. ANB provides the air interface between the control plane protocol terminations and the user plane towards the autonomous Human Machine Interface (HMI). The interfaces of network are built on IP protocols. ANB is equipped with different MAC protocols to deliver different types of data information. The SG/GGSN act as a limited anchor for the mobility service for transmitting and receiving packet rates from and to the requester and cloud to serve the HMI request. Mobility Management Entity (MME) is an entity to afford signaling only, and later, the user packets of the IP do not pass over the MME. SDN controller allows administrators to manage network services through abstraction of lower-level functionality. A cloud server is a logical server that is built, hosted, and delivered through a cloud computing platform over the Internet. Cloud servers exhibit and possess similar capabilities and functionality to a usual server but are accessed remotely on-demand from a cloud service provider. The cloud server provides computation, data access, software, services, and storage that do not require service requester's knowledge about the physical location and configuration of the system that deliver services.

As shown in Fig. 2. Service-level agreement (SLA) is the negotiation between the cloud server and service requesters for maintaining the QoS. Some metrics that SLAs may specify in the $5 \mathrm{CVN}$ are as follows:

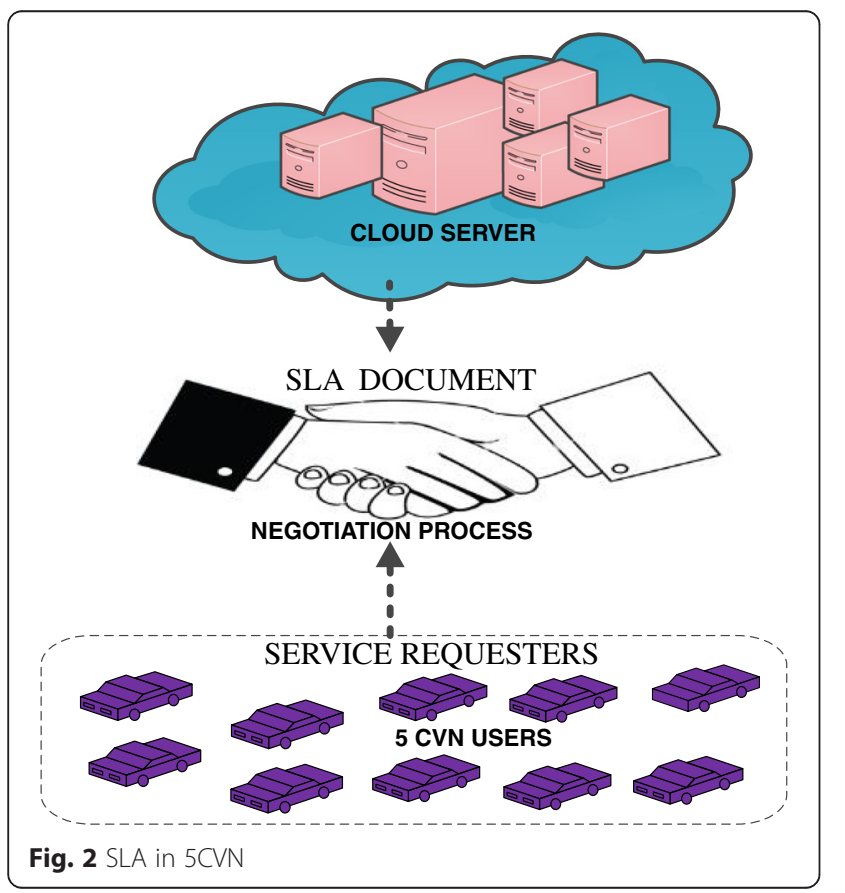

- The number of $5 \mathrm{CVN}$ users that can be served simultaneously by the cloud.

- The particular performance benchmark to which actual performance to be compared periodically.

- Advance notification about the network changes.

- Help desk response time for different classes of problems and dial-in access accessibility.

\section{$35 \mathrm{CVN}$-adroit algorithm}

The $5 \mathrm{CVN}$-adroit algorithm discovers an optimal gateway and ANB in the network layer to route the request/ response from/to the requesters and the cloud using $5 \mathrm{G}$ technologies. An ingress vehicle (service requester vehicle) coverage area is IEEE $802.11 \mathrm{p}$ wireless transmission range. As expressed in Eq. 1, the coverage area of an ingress vehicle is determined as follows:

$$
C_{\text {area }}=\mathrm{TR}_{\max }(1-\alpha)
$$

where $\mathrm{TR}_{\max }$ denotes the maximum IEEE 802.11p transmission range and $\alpha$ reflects the wireless channel fading conditions of the ingress vehicle location. For instance, $\alpha$ can be set to small values in environments when there are no major obstacles and takes high values in urban areas with tall buildings. A mapping function between the geographical locations and the values of $\alpha$ can be provided by using position system such as GPS and Galileo. As illustrated in Algorithm 1, the $5 \mathrm{CVN}$-adroit algorithm consists of two key processes such as metric-based gateway selection and metric-based ANB selection.

\subsection{Metric-based gateway selection}

The ingress vehicle dynamically scans for the neighbor vehicle in the coverage area. Then, the ingress vehicle will send the beacon message to each neighbor vehicle and get the updated information periodically about the signal strength, number of packets in the buffer of the neighbor vehicle, and distance between ingress vehicle and neighbor vehicle. Based on the above periodic messages, the ingress vehicle calculates the fitness value for each neighbor vehicle, as given in Eq. 2. If an ingress vehicle does not receive reply beacon messages from the specific neighbor vehicle during a certain time period, then the link is considered down. The neighbor vehicle is discarded. The fitness value ( $\mathrm{fv}$ ) for gateway selection (GS) is computed as follows:

$$
\begin{aligned}
\mathrm{fv}(\mathrm{GS}) & =\frac{S_{\mathrm{str}}}{\mathrm{NP} * D_{t, i}} \quad \forall n, n \\
& =1,2,3, \ldots \ldots, r
\end{aligned}
$$

where " $\mathrm{fv}(\mathrm{GS})$ " is the fitness value for gateway selection, " $\mathrm{S}_{\text {str }}$ " is the signal strength of the neighbor vehicle in terms of $\mathrm{dBm}$, "Np" is the number of packets in the buffer of the neighbor vehicle, " $D_{t, i}$ " is the distance between 
the ingress vehicle and the neighbor vehicle in terms of meter, and " $r$ " is the maximum number of the neighbor vehicles. Among all possible neighbor vehicles (NV) $\mathrm{NV} \in F \subset C_{\text {area }}$, we want to determine a gateway $\left(G_{w}\right)$ that optimizes (maximum) the function fv:

$$
G_{w}=\arg \max _{\mathrm{NV} \in F \subset C_{\text {area }}} \mathrm{fv}(\mathrm{GS})
$$

where $F$ is the feasible solution. As expressed in Eq. 3, among the neighbor vehicles, the vehicle which has the maximum fitness value is chosen as the gateway. The gateway activates its interface in order to communicate with the ANB. Then, the request from the ingress vehicle is transmitted to the gateway. The chosen gateway broadcasts periodic gateway advertisement (GWADV) messages within its coverage area up to time-to-live (TTL) value.

\subsubsection{Selecting on-hand gateway}

The new ingress vehicle checks for an on-hand (existing) gateway in its coverage area. If there is a gateway in its coverage area, then the new ingress vehicle will transmit the service request to an on-hand gateway and then go to the metric-based gateway selection.

\subsection{Metric-based ANB selection}

The range is distinct as the coverage area of the ANB. The ANB broadcasts periodic ANB advertisement (ANBADV) messages within its range (R). The gateway dynamically checks for the ANB to transmit the service request.

\subsubsection{Case 1}

If there is only one ANB, from the gateway, the service request is stanchly transmitted to the ANB.

\subsubsection{Case 2}

If there are no ANBs, the gateway dynamically scans for the neighbor vehicle towards its moving direction within its coverage area. Then, the gateway will send the beacon message to the each neighbor vehicle in the list. It gets the updated information periodically about the signal strength of the neighbor vehicle, number of packets in the buffer of the neighbor vehicle, and distance between the gateway and neighbor vehicle. Based on the above periodic messages, the gateway calculates the fitness value for each neighbor vehicle, as given in Eq. 4. If a gateway does not receive reply beacon messages from the specific neighbor vehicle during a certain time period, then the link is considered down. The neighbor vehicle is discarded. The fitness value (fv) for subsequent gateway selection (SGS) is computed as follows:

$$
\begin{aligned}
\mathrm{fv}(\mathrm{SGS}) & =\frac{S_{\text {str }}}{\mathrm{NP} * D_{q, e}} \quad \forall n, n \\
& =1,2,3, \ldots \ldots, s
\end{aligned}
$$

where " $\mathrm{fv}(\mathrm{SGS})$ " is the fitness value for subsequent gateway selection, " $\mathrm{S}_{\text {str }}$ " is the signal strength of the neighbor vehicle in terms of $\mathrm{dBm}$, "Np" is the number of packets in the buffer of the neighbor vehicle, " $\mathrm{D}_{\mathrm{q}, \mathrm{e}}$ " is the distance between the gateway and the neighbor vehicle in terms of meter, and " $\mathrm{s}$ " is the maximum number of the neighbor vehicles. Among all possible neighbor vehicles $(\mathrm{NV}) \mathrm{NV} \in F \subset C_{\text {area }}$, we want to determine a subsequent gateway $\left(\mathrm{SG}_{\mathrm{w}}\right)$ that optimizes (maximum) the function $\mathrm{fv}$ :

$$
\mathrm{SG}_{\mathrm{w}}=\arg \max _{\mathrm{NV} \in F \subset C_{\text {area }}} \mathrm{fv}(\mathrm{SGS})
$$

where $F$ is the feasible solution. As expressed in Eq. 5, among the neighbor vehicles, the vehicle which has the maximum fitness value is chosen as the subsequent gateway. From the gateway, the service request is transmitted to the subsequent gateway and then goes to the metricbased ANB selection. Case 1 and case 2 are the worst cases.

\subsubsection{Case 3}

If there are two or more ANBs, the gateway will send the beacon message to each neighbor ANB, gets the upto-the-minute information about the number of packets in the buffer of the ANB and the distance between the gateway and ANB, and calculates a fitness value for each ANB, as given in Eq. 6 . If the gateway does not receive beacon messages from the specific neighbor ANB during a certain time period, then the link is considered down. The ANB is discarded. The fitness value (fv) for ANB selection (ANBS) is calculated as follows:

$$
\begin{aligned}
\mathrm{fv}(\mathrm{ANBS}) & =\mathrm{NPB}+D_{g, a} \quad \forall n, n \\
& =1,2,3, \ldots \ldots, w
\end{aligned}
$$

where " $\mathrm{fv}(\mathrm{ANBS})$ " is the fitness value for ANB selection, "NPB" is the number of packets in the buffer of the ANB, " $\mathrm{D}_{\mathrm{g}, \mathrm{a}}$ " is the distance between the gateway and ANB, and " $w$ " is the maximum number of ANBs. Among all possible ANBs, $\mathrm{ANB} \in F \subset R$, we want to determine an ANB that optimizes (minimum) the function fv:

$$
\mathrm{OANB}=\arg \min _{\mathrm{ANB} \in \mathrm{FS} \subset R} f \mathrm{v}(\mathrm{ANBS})
$$

where FS is the feasible solution. As expressed in Eq. 7, among the ANBs, the ANB, which has the minimum fitness value, is chosen as an optimal ANB (OANB). From the gateway, the request is transmitted to the optimal ANB.

The computing enhanced optimal ANB checks for the services in the local cloud as per the user requirement. If the service is found, the optimal ANB transmits the service to the requester. Else from the optimal ANB, the service request is transmitted to the cloud server via MME, SG/ GGSN, and SDN controller. Service reply from the cloud server to the service requester follows the same request path. 


\begin{tabular}{|c|c|}
\hline \multicolumn{2}{|l|}{$\begin{array}{l}\text { Source: Ingress Vehicle } \\
\text { Destination: ANB or else Cloud Server } \\
\text { Input: Service Request } \\
\text { Output: Response from the ANB or cloud server } \\
\text { Process: Route Discovery }\end{array}$} \\
\hline 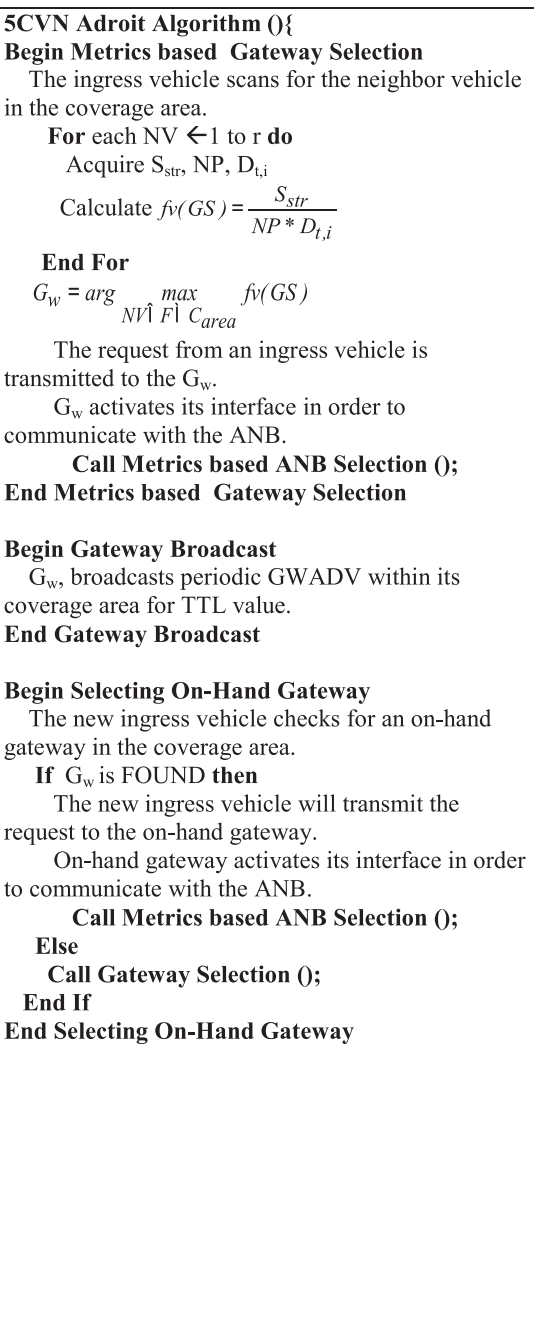 & 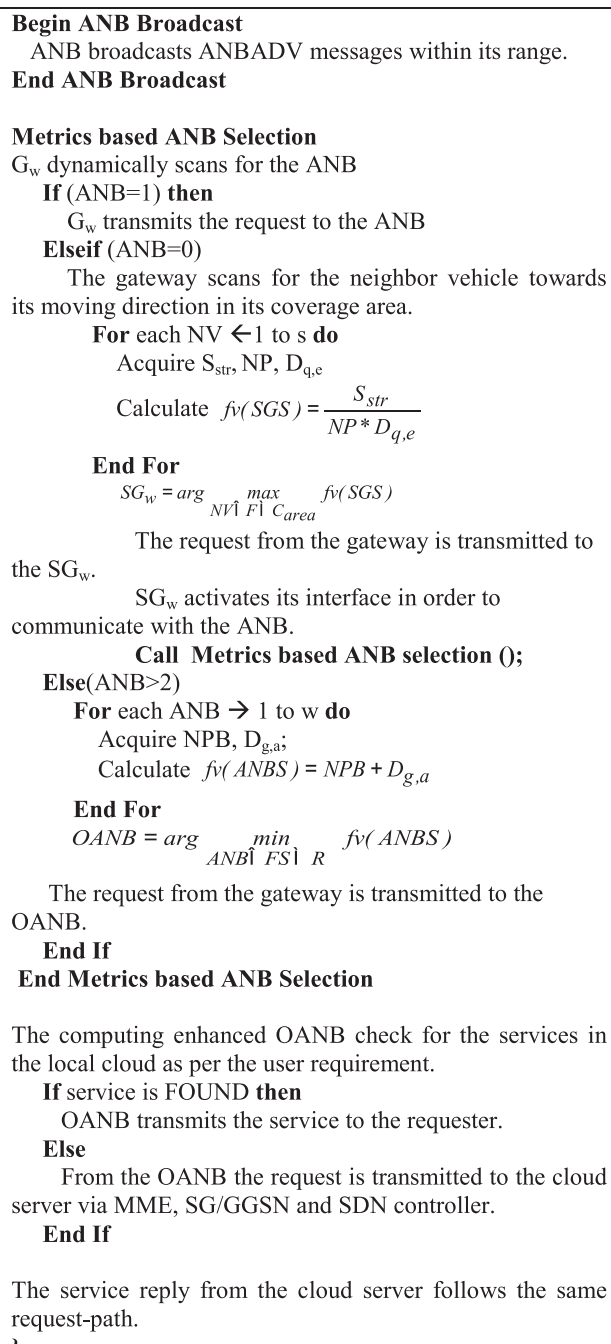 \\
\hline
\end{tabular}

\section{Simulation results and analysis}

We implemented the $5 \mathrm{CVN}$-adroit algorithm and the existing techniques on network simulator (NS2) and used the topology of the network generated by Simulation of Urban Mobility (SUMO). SUMO is the microscopic, open source, and multimodal traffic simulator. The simulation allows to address a large set of traffic management topics. Each vehicle is modeled explicitly and has an own route and travel independently through the network. Here, SUMO is coupled to a communication network simulator using a middleware (VSimRTI). The V2X Simulation Runtime Infrastructure (VSimRTI) is a framework which enables the groundwork and execution of V2X simulations. The easier integration and exchange of simulators enables the replacement of the most relevant simulators for a realistic presentation of traffic, communication, and the execution of V2X applications. The NuoDB is a cloud database and offers a well-off SQL implementation and factual ACID transactions. Table 1 illustrates the characteristics of the environment in which the simulation is experimented. In the simulation, the request generation speeds remnants at five packets per second when the vehicle density changes.

In our existing work, we have proposed Optimized Heuristic Buffer-based Routing (OHBR) which focuses on proficient routing based on the buffer size and heuristic 
Table 1 Simulation parameters

\begin{tabular}{ll}
\hline Simulation parameters & Value \\
\hline Network simulator & NS2 \\
Traffic simulator & SUMO-0.15.0 \\
Cloud server & NuODB \\
Number of nodes & 500 \\
Number of ANB & 18 \\
MAC protocol & IEEE 802.11p \\
Real-time environment model & Rayleigh fading environment \\
Connection type & CBR or UDP \\
IP & IPV6 \\
Packet size & $64 \mathrm{kB}$ \\
CBR interval & $10 \mathrm{~s}$ \\
Nodes speed & $20-160 \mathrm{~km} / \mathrm{h}$ \\
Gateway broadcast timer & $1 \mathrm{~s}$ \\
Antenna type & Antenna/omni directional antenna \\
\hline
\end{tabular}

fitness function, in order to reduce the packet loss, retransmission, gridlock, protocol overhead, and delay in VMesh milieu [5]. In this work, we do not consider the base station selection which results in data traffic at the base station. The data traffic results in delay, packet loss, and packet collisions. Abderrahim et al. proposed a mechanism and named as Clustering-based Multi-metric adaptive mobile Gateway Management (CMGM) in which vehicles are dynamically clustered according to different related metrics such as UMTS received signal strength, direction of vehicles, and vehicle to vehicle distance [17]. However, the cluster mechanism leads to confusion in electing the cluster head. And as well in CMGM fixing, the gateway candidate progression is complex which results in a delay in gateway discovery mechanism. Kaouther et al. have proposed efficient and scalable LocVSDPs for VNs. The proposed protocols have vehicles provided with an efficient mechanism to locate service providers and how to reach them (routing) simultaneously [18]. However, in LocVSDPs for VNs, clustering leads to confusion in electing the cluster head and it will increase the routing overhead and delay. The performance of the proposed 5CVN-adroit algorithm and existing OHBR, 3G-CMGM, and LocVSDPs are assessed in terms of the packet delivery ratio, average response time, routing overhead ratio, and packet collision ratio. The intricate descriptions of these indexes are as follows:

Packet Delivery Ratio (PDR): PDR is defined as the ratio between the number of responses received by the source to the number of requests sent.

Average Response Time (ART): ART is the average difference between the time a request packet is originated by an application and the time the response packet is received.

Routing Overhead Ratio (ROR): ROR is defined as the ratio between the total number of bytes of control packets and the cumulative size of data packets which are delivered to the destinations to the total number of control packets and the cumulative size of data packets sent.

Packet Collision Ratio (PCR): The packet collision ratio is defined as the number of packets collided to the number of packets transmitted. This gives an indication of the loading of the network.

Table 2 depicts the simulation results obtained by the proposed $5 \mathrm{CVN}$-adroit algorithm (A), existing OHBR $(\mathrm{O})$, 3G-CMGM (C), and LocVSDPs (L) for different request generation speed (RGS) and vehicle density (VD). Based on the obtained results, we calculate the PDR, ART, RO, and PCR. In our existing work Optimized Heuristic Bufferbased Routing (OHBR), we do not consider the base station pool and the base station selection which results in data traffic at the base station when the RGS and VD rises. This results in delay, packet loss, and packet collisions. Consequently, PDR and RO decrease, and ART and PCR increase. In existing 3G-CMGM, the cluster mechanism leads to confusion in electing the cluster head. It results in a decrease in the PDR and RO and increase in the ART and PCR. In addition, in CMGM fixing, the gateway candidate progression is complex which results in a delay in gateway discovery mechanism. The existing LocVSDPs relies on roadside routers. The roadside router has to be planned and deployed on the road side. Consequently, the deployment cost is high. LocVSDPs is also the clusterbased approach. The cluster creates confusion in the routing process, results in the diminishing of the PDR and RO, and rises in the ART and PCR. However, in the proposed mechanism by using the $5 \mathrm{G}$ technology components, adroit algorithm, and local cloud at ANB, the VMesh user can enjoy with the minimum delay and maximum delivery ratio.

The impact of various RGS on PDR is illustrated in Fig. 3. It can be deduced from this figure that PDR decreases as the RGS rises. Higher RGS causes more network topology changes and more link failures and thereby PDR decreases accordingly. Owing to the introduction of gateway and ANB selection based on the relevant metrics, the probability of disconnections between platoons shows an improvement in the $5 \mathrm{CVN}$-adroit algorithm over to the OHBR, 3G-CMGM, and LocVSDPs. Hence, the PDR of the $5 \mathrm{CVN}$-adroit algorithm outperforms the existing techniques. As depicted in Fig. 4, the ART of OHBR, 3GCMGM, LocVSDPs, and 5CVN-adroit algorithm increases when the number of request increases. Higher RGS generates more network topology changes and more delay. In 
Table 2 Simulation results

\begin{tabular}{|c|c|c|c|c|c|c|c|c|c|c|c|c|c|c|c|c|}
\hline \multirow[t]{2}{*}{ RGS } & \multicolumn{4}{|l|}{ PDR } & \multicolumn{4}{|l|}{ ART } & \multicolumn{4}{|l|}{$\mathrm{RO}$} & \multicolumn{4}{|l|}{ PCR } \\
\hline & A & 0 & $C$ & $\mathrm{~L}$ & A & 0 & $C$ & $\mathrm{~L}$ & A & 0 & $C$ & $\mathrm{~L}$ & A & 0 & $C$ & $\mathrm{~L}$ \\
\hline 5 & 0.96 & 0.88 & 0.76 & 0.5 & 0.04 & 0.07 & 0.09 & 0.4 & 100 & 92 & 80 & 50 & 0.02 & 0.1 & 0.3 & 0.5 \\
\hline 50 & 0.94 & 0.8 & 0.76 & 0.5 & 0.05 & 0.08 & 0.1 & 0.5 & 94 & 80 & 76 & 50 & 0.02 & 0.1 & 0.32 & 0.58 \\
\hline 100 & 0.92 & 0.77 & 0.69 & 0.46 & 0.06 & 0.09 & 0.2 & 0.7 & 92 & 77 & 69 & 46 & 0.02 & 0.1 & 0.36 & 0.6 \\
\hline 150 & 0.9 & 0.77 & 0.67 & 0.46 & 0.06 & 0.1 & 0.2 & 0.8 & 90 & 77 & 67 & 46 & 0.02 & 0.12 & 0.37 & 0.6 \\
\hline 200 & 0.89 & 0.67 & 0.61 & 0.45 & 0.07 & 0.2 & 0.3 & 0.9 & 89 & 67 & 65 & 45 & 0.024 & 0.12 & 0.4 & 0.62 \\
\hline 250 & 0.88 & 0.66 & 0.6 & 0.44 & 0.07 & 0.3 & 0.4 & 1.1 & 88 & 66 & 60 & 44 & 0.026 & 0.13 & 0.4 & 0.7 \\
\hline 300 & 0.85 & 0.62 & 0.57 & 0.42 & 0.07 & 0.4 & 0.6 & 1.2 & 85 & 62 & 57 & 42 & 0.028 & 0.17 & 0.4 & 0.8 \\
\hline 350 & 0.85 & 0.6 & 0.5 & 0.42 & 0.07 & 0.5 & 0.9 & 1.3 & 80 & 55 & 45 & 42 & 0.035 & 0.17 & 0.45 & 0.85 \\
\hline \multirow[t]{2}{*}{ VD } & PDR & & & & ART & & & & $\mathrm{RO}$ & & & & PCR & & & \\
\hline & A & 0 & $C$ & L & A & 0 & $C$ & L & A & 0 & $C$ & L & A & 0 & $C$ & L \\
\hline 25 & 0.96 & 0.88 & 0.72 & 0.6 & 0.02 & 0.05 & 0.06 & 0.1 & 100 & 88 & 72 & 60 & 0.01 & 0.03 & 0.04 & 0.1 \\
\hline 250 & 0.92 & 0.86 & 0.7 & 0.6 & 0.03 & 0.06 & 0.07 & 0.15 & 96 & 86 & 70 & 60 & 0.01 & 0.034 & 0.062 & 0.1 \\
\hline 500 & 0.92 & 0.82 & 0.7 & 0.6 & 0.05 & 0.07 & 0.08 & 0.2 & 96 & 82 & 70 & 60 & 0.01 & 0.035 & 0.07 & 0.1 \\
\hline 750 & 0.92 & 0.81 & 0.56 & 0.53 & 0.06 & 0.08 & 0.1 & 0.25 & 95 & 81 & 65 & 53 & 0.02 & 0.036 & 0.075 & 0.12 \\
\hline 1000 & 0.91 & 0.81 & 0.45 & 0.42 & 0.06 & 0.08 & 0.2 & 0.3 & 95 & 81 & 60 & 42 & 0.02 & 0.038 & 0.08 & 0.13 \\
\hline 1250 & 0.8 & 0.72 & 0.44 & 0.32 & 0.068 & 0.09 & 0.3 & 0.35 & 90 & 72 & 55 & 32 & 0.02 & 0.039 & 0.088 & 0.13 \\
\hline 1500 & 0.8 & 0.6 & 0.4 & 0.3 & 0.08 & 0.1 & 0.4 & 0.45 & 90 & 72 & 50 & 30 & 0.02 & 0.04 & 0.093 & 0.14 \\
\hline 1750 & 0.8 & 0.6 & 0.4 & 0.3 & 0.08 & 0.2 & 0.5 & 0.6 & 90 & 72 & 50 & 30 & 0.03 & 0.044 & 0.095 & 0.15 \\
\hline
\end{tabular}

existing techniques, the selected gateways and the base station will be overloaded with packets, some of which will be left over, finally impacting the packet loss and retransmission, which will increase the response time. In the $5 \mathrm{CVN}$-adroit algorithm, as the gateway and the ANB are chosen based on the minimum number of packets in the buffer, it will decrease the processing delay. In addition, in the $5 \mathrm{CVN}$-adroit algorithm, the next-hop gateway and the

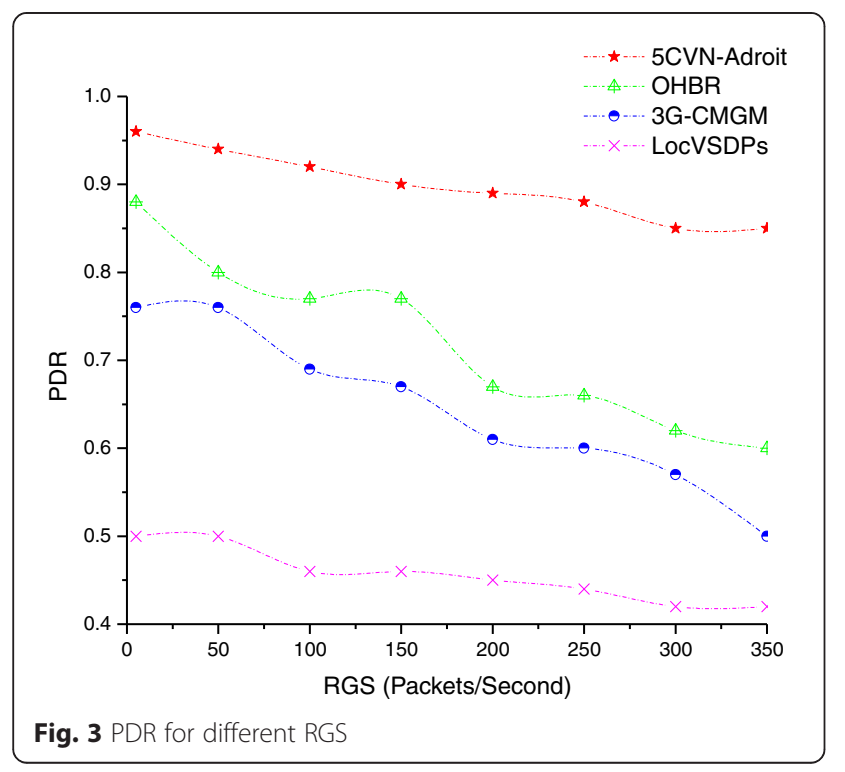

ANB are picked based on the fitness value and reserved ahead before ingress vehicle transmitting the request. As a consequence, the ART of the $5 \mathrm{CVN}$-adroit algorithm outperforms the ART of OHBR, 3G-CMGM and LocVSDPs. Accordingly, we have compared the ROR performance of the 5CVN-adroit algorithm, OHBR, 3G-CMGM, and LocVSDPs with RGS varying in Fig. 5. It can be figured out from this figure that ROR decreases as the RGS rises.

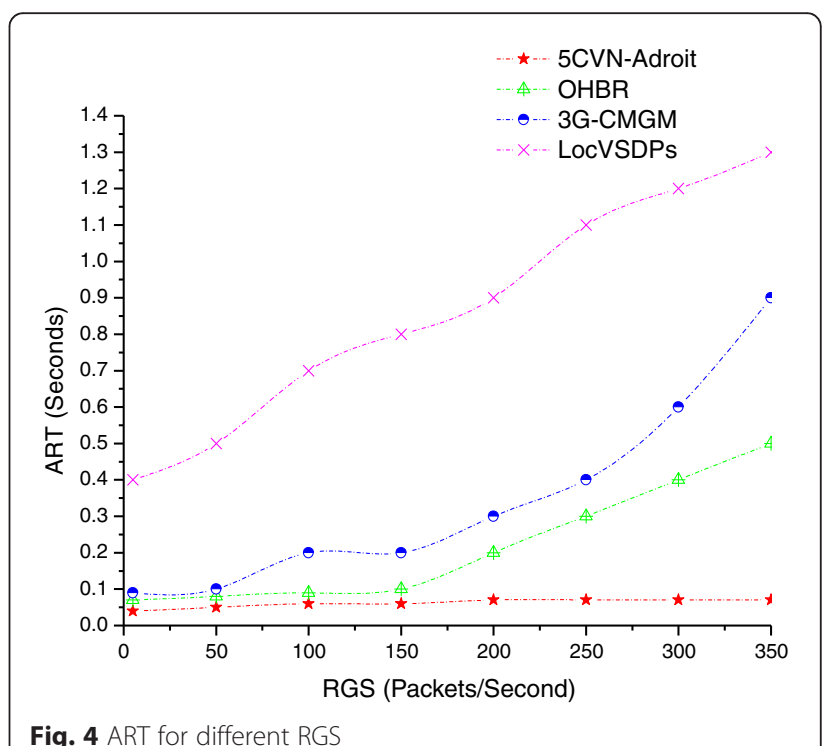




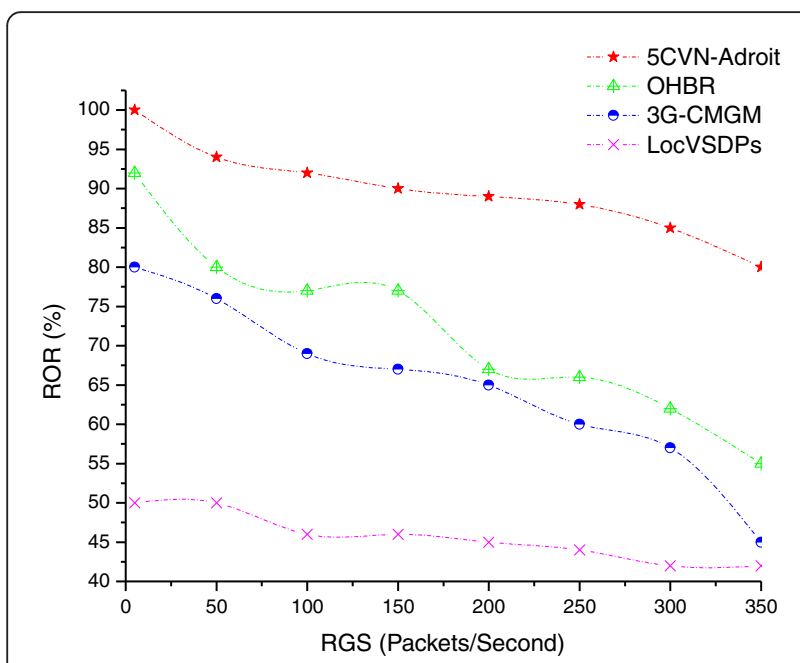

Fig. 5 ROR for different RGS

By virtue of gateway selection, the $5 \mathrm{CVN}$-adroit algorithm is more likely to find a route from source to destination without any confusion in the routing process. Thus, the $5 \mathrm{CVN}$-adroit algorithm outperforms the ROR of OHBR, 3G-CMGM, and LocVSDPs in terms of higher routing overhead ratio. It can be deduced from Fig. 6 that PCR of OHBR, 3G-CMGM, LocVSDPs, and 5CVN-adroit algorithm increases as the RGS increases. This result is rational and illustrates that high data rate may easily inundate the service channel of IEEE 802.11p since no differentiated service is being used. Compared to OHBR, 3G-CMGM, and LocVSDPs, the $5 \mathrm{CVN}$-adroit algorithm shows the superlative PCR owing to its routing selection concern which combines the delay estimation and path connectivity together.

The influence of vehicle density on PDR of the $5 \mathrm{CVN}$ adroit algorithm, OHBR, 3G-CMGM, and LocVSDPs is

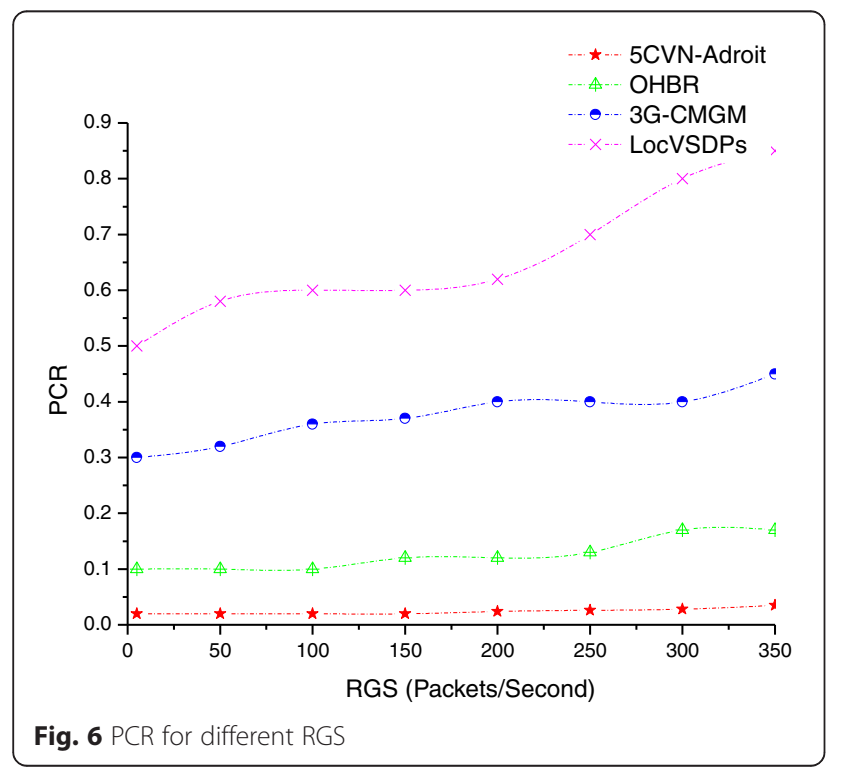

plotted in Fig. 7. It is worth stressing that the impact of vehicle density on PDR is more apparent than that of RGS. The PDR of OHBR, 3G-CMGM, LocVSDPs, and 5CVNadroit algorithm generally shows a diminishing trend in the growth of vehicle density. However, a large vehicle density will introduce more collisions and retransmissions on the IEEE 802.11p MAC layer. Thus, the OHBR, 3GCMGM, LocVSDPs, and 5CVN-adroit algorithm experience a dropping PDR when vehicle density increases. Due to the usage of real connected paths in $5 \mathrm{CVN}$-adroit algorithm between source and destination, it can easily tolerate MAC collisions, thus still providing better PDR when vehicle density is more. From Fig. 8 , it can be deduced that with the continuous increase of vehicle density, the ART of OHBR, 3G-CMGM, LocVSDPs, and 5CVN-adroit algorithm also increases. Higher density generates more network topology changes and more delay. By selecting the qualified gateway and ANB based on relevant metrics, ART of adroit algorithm outperforms the ART of existing techniques. In addition, by using the local cloud in computing enhanced ANB, the ART of 5CVN-adroit algorithm outperforms the existing techniques. As shown in Fig. 9, the ROR of the 5CVN-adroit algorithm, OHBR, 3GCMGM, and LocVSDPs decreases when the vehicle density increases. The existing techniques create confusions in the routing process; hence, it will decrease the routing overhead ratio. To avoid the confusion in adroit algorithm, quantified gateway and ANB has been selected. In addition, the next-hop gateway and ANB is picked and reserved ahead; that causes less routing overhead. Therefore, the ROR of the proposed scheme is high compared to the existing techniques. The $5 \mathrm{CVN}$-adroit algorithm has some advantage in the aspects of ROR by considering important path selection factors such as link availability and link quality,

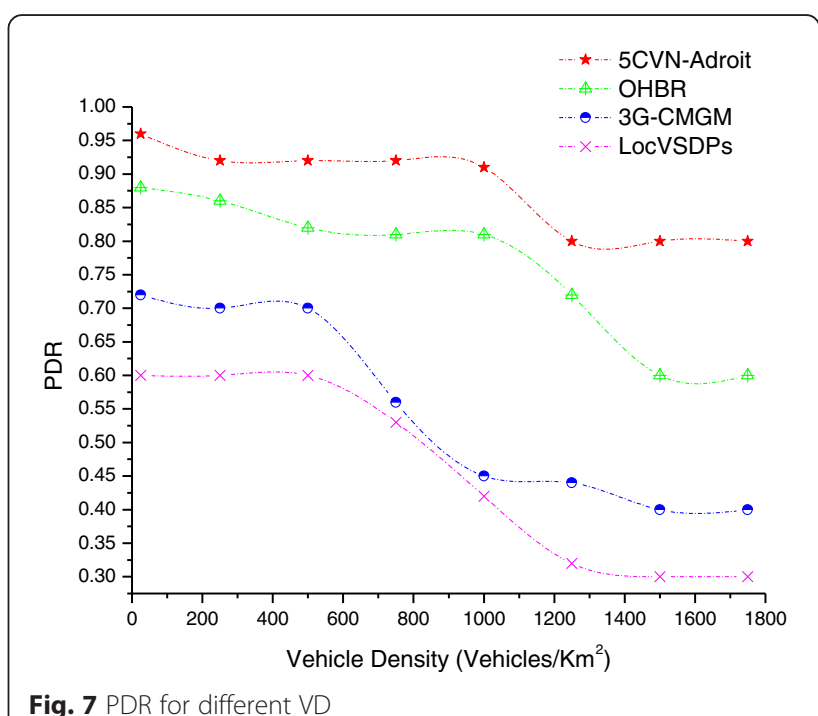




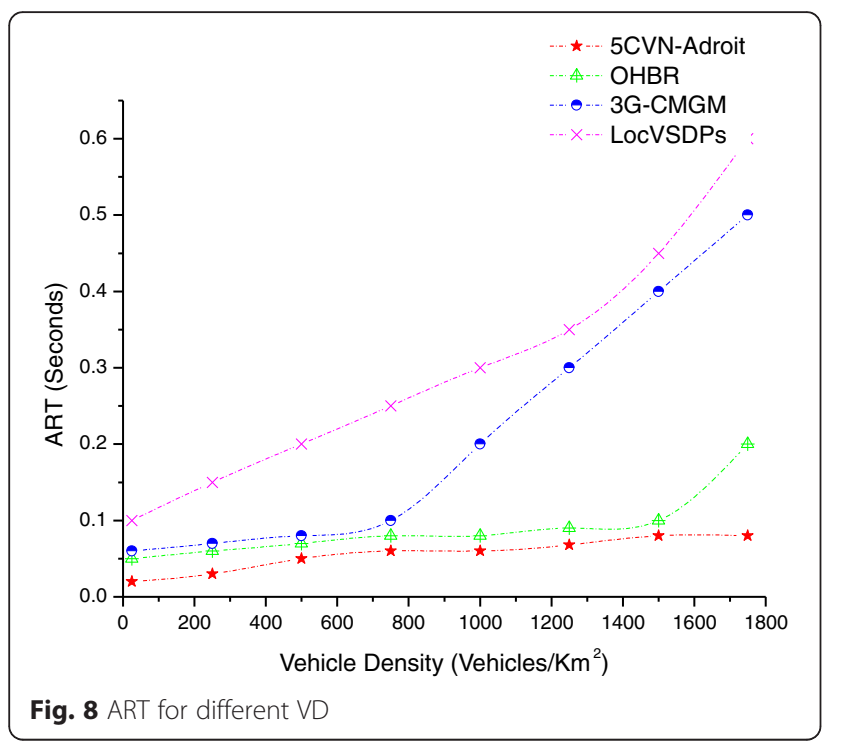

which is of great importance to select a better route. Thus, the 5CVN-adroit algorithm shows better reliability and scalability. PCR is affected by different parameters such as vehicle density and traffic load. The results presented in Fig. 10 suggest that the proposed $5 \mathrm{CVN}$-adroit algorithm is not affected as much as existing techniques. The increase in vehicle density brings more collisions on MAC. The reason behind is that more retransmission and collision causes the routing layer to use more beacons. The $5 \mathrm{CVN}$-adroit algorithm reduces the retransmission and collision by introducing an ANB selection mechanism among an ANB pool. Thus, the PCR of adroit algorithm outperforms the existing techniques.

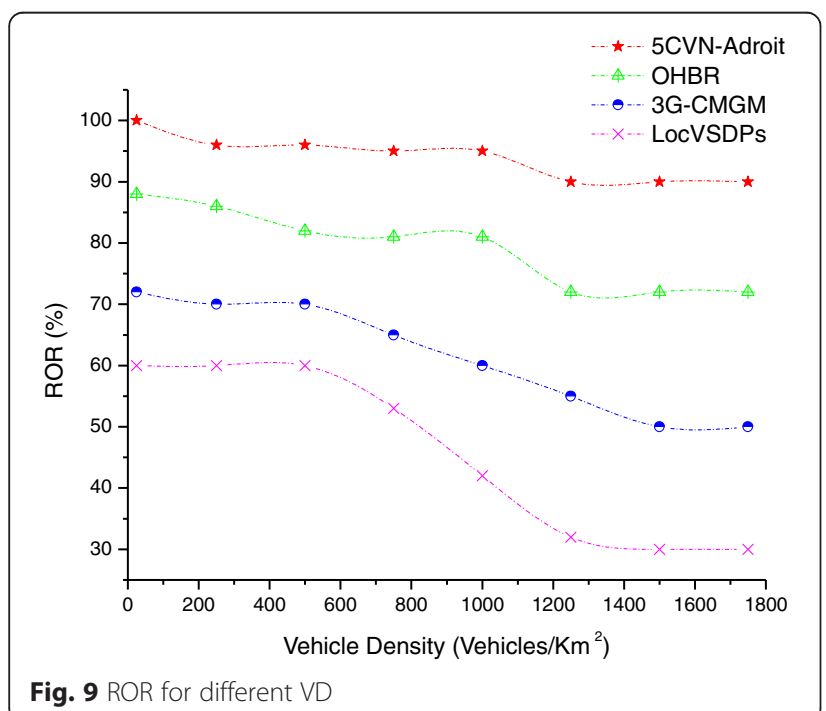

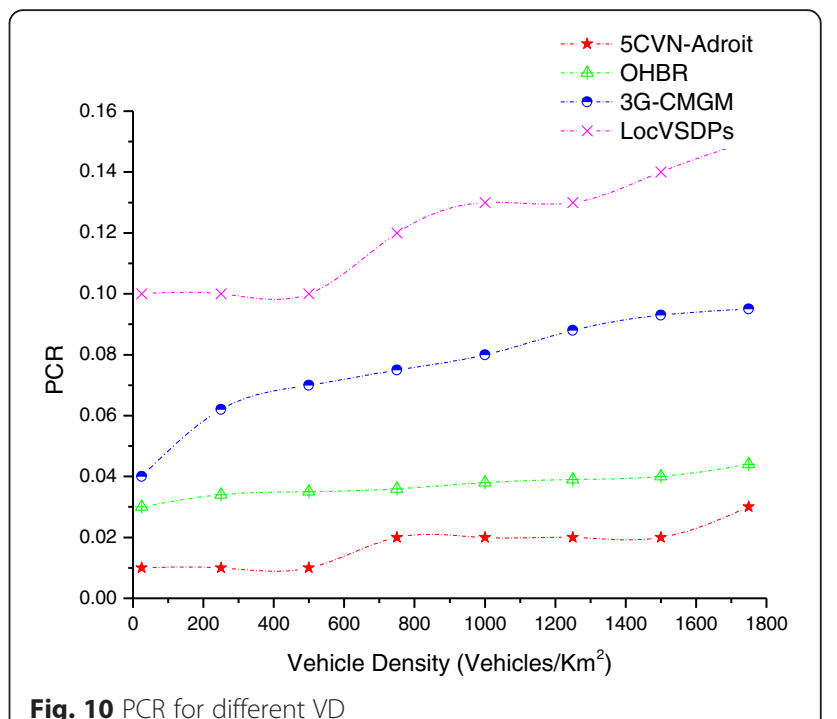

Fig. 10 PCR for different VD

\section{Conclusions}

The proposed $5 \mathrm{CVN}$-adroit algorithm affords high performance, globally optimized load balancing and network usage increased from $30 \%$ to over $90 \%$. In this paper, we initiate an architecture that amalgamates 5G SDN, cloud, and VMesh networks, which offers advanced network services and apps at software speed for the users. The simulation results show that our $5 \mathrm{CVN}$-adroit algorithm is very suitable for real-time applications and outperforms the existing techniques on packet delivery ratio, average response time, routing overhead ratio, and packet collision ratio. However, there is still much research needed to fully exploit the potential of $5 \mathrm{CVN}$. This paper may be giving a good platform to motivate the researchers for a better outcome of different types of problems in next generation networks.

\section{Competing interests}

The authors declare that they have no competing interests.

\section{About the authors}

P. Dharanyadevi is a research scholar in the Department of Information and Communication Engineering, Anna University, Chennai, India. She finished her master degree at Pondicherry University. Her research areas include web services, service oriented architecture, ad hoc milieus, pervasive computing, context aware computing, and cloud computing. She has published many research papers in International Journals and Conferences.

Dr. Venkatalakshmi Krishnan is working as Assistant Professor and Head of the Department in the Department of Electronics and Communication Engineering, Anna University Tindivanam Campus, Tindivanam, India. Her research areas include networks, pattern recognition, evolutionary protocols, and multispectral data fusion. She has published many research papers in International Journals and Conferences. She is an eminent reviewer in most of the reputed journals.

\section{Author details}

${ }^{1}$ Information and Communication Engineering, Anna University, Chennai, India. ${ }^{2}$ Electronics and Communication Engineering, Anna University Tindivanam Campus, Tindivanam, India. 
Received: 26 January 2016 Accepted: 13 March 2016

Published online: 31 March 2016

\section{References}

1. P Dharanyadevi, K Venkatalakshmi, Potent gateway selection algorithm for integrated 3G-VMesh milieu. World Appl. Sci. J. 33, 1228-1233 (2015). doi:10.5829/idosi.wasj.2015.33.07.243

2. Y-S Chen, C-S Hsu, C-H Cheng, Network mobility protocol for vehicular ad hoc networks. Int. J. Commun. Syst. 27, 3042-3063 (2014)

3. W Xiaonan, Q Huanyan, Constructing a VANET based on cluster chain. Int. J. Commun. Syst. 27, 2497-2517 (2014)

4. H. Moustafa, Y. Zhang. Vehicular networks_-techniques, standards, and applications. CRC Press. 2009.

5. P Dharanyadevi, K Venkatalakshmi, Optimized heuristic buffer-based routing for VMesh milieu. Aust. J. Basic Appl. Sci. 9, 386-390 (2015)

6. P Dharanyadevi, K Venkatalakshmi, Reliable and scalable routing protocol (RSRP) for multimedia data transmission in vehicular mesh milieu. Int. J. Appl. Eng. Res. 9, 19935-19946 (2015)

7. $\quad$ PK Agyapong, M Iwamura, D Staehle, W Kiess, A Benjebbour, Design considerations for a $5 \mathrm{G}$ network architecture. IEEE Commun. Mag. 52, 65-75 (2014). doi:10.1109/MCOM.2014.6957145

8. C-X Wang, Cellular architecture and key technologies for $5 \mathrm{G}$ wireless communication networks. IEEE Commun. Mag. 52, 122-130 (2014). doi:10.1109/MCOM.2014.6736752

9. E Hans-Joachim, S Patrick, T Riccardo, System design for $5 \mathrm{G}$ converged networks, 2015, pp. 391-396. doi:10.1109/EuCNC.2015.7194105. European Conference on Networks and Communications (EuCNC)

10. Z Zhang, Full duplex techniques for $5 G$ networks: self-interference cancellation, protocol design, and relay selection. IEEE Commun. Mag. 53, 128-137 (2015). doi:10.1109/MCOM.2015.7105651

11. Z Zhang, Large-scale MIMO based wireless backhaul in $5 \mathrm{G}$ networks. IEEE Commun. Mag. 22, 58-66 (2015). doi:10.1109/MWC.2015.7306538

12. Y Mao, Software-defined and virtualized future mobile and wireless networks: a survey. MONET 20, 4-18 (2015). doi:10.1007/s11036-014-0533-8

13. Y Niu, A survey of millimeter wave communications (mmWave) for $5 \mathrm{G}$ opportunities and challenges. Wirel. Netw. 21, 2657-2676 (2015). doi:10.1007/s11036-014-0533-8

14. R Guerzoni, R Trivisonno, D Soldani, SDN-based architecture and procedures for 5 G networks, 2014. doi:10.1007/s11036-014-0533-8. 1st International Conference on $5 \mathrm{G}$ for Ubiquitous Connectivity (5GU)

15. H Nam, Intelligent content delivery over wireless via SDN, 2015, pp. 2185-2190. IEEE Wireless Communications and Networking Conference (WCNC): Track 4 - Services, Applications, and Business

16. P Ameigeiras, JJ Ramos-Munoz, L Schumacher, J Prados-Garzon, J Navarro-Ortiz, JM Lopez-Soler, Link-level access cloud architecture design based on SDN for 5G networks. IEEE Netw. 29, 24-31 (2015). doi:10.1109/MNET.2015.7064899

17. B Abderrahim, A Benslimane, T Taleb, R Sivaraj, Dynamic clustering-based adaptive mobile gateway management in integrated VANET-3G heterogeneous wireless networks. IEEE J. Sel. Areas Commun. 29(3), 559-570 (2011)

18. K Abrougui, A Boukerche, RWN Pazzi, Design and evaluation of contextaware and location-based service discovery protocols for vehicular networks. IEEE Trans. Intell. Transp. Syst. 12(3), 717-735 (2011)

\section{Submit your manuscript to a SpringerOpen ${ }^{\mathcal{O}}$ journal and benefit from:}

- Convenient online submission

- Rigorous peer review

- Immediate publication on acceptance

- Open access: articles freely available online

- High visibility within the field

- Retaining the copyright to your article

Submit your next manuscript at $\gg$ springeropen.com 